\title{
Exploring How a Co-dependent Tangible Tool Design Supports Collaboration in a Tabletop Activity
}

\author{
Min Fan ${ }^{1}$, Alissa N. Antle ${ }^{1}$, Carman Neustaedter ${ }^{1}$, Alyssa F. Wise ${ }^{2}$ \\ ${ }^{1}$ School of Interactive Arts + Technology ${ }^{2}$ Faculty of Education \\ Simon Fraser University \\ 250-13450 102 Avenue \\ Surrey, B.C. Canada \\ V3T OA3 \\ Simon Fraser University \\ 250 -13450 102 Avenue \\ Surrey, B.C., Canada \\ V3T 2W1 \\ [minf, aantle, carman_neustaedter, afw3]@sfu.ca
}

\begin{abstract}
Many studies suggest that tangibles and digital tabletops have potential to support collaborative interaction. However, previous findings show that users often work in parallel with such systems. One design strategy that may encourage collaboration rather than parallel use involves creating a system that responds to codependent access points in which more than one action is required to create a successful system response. To better understand how co-dependent access points support collaboration, we designed a comparative study with 12 young adults using the same application with a co-dependent and an independent access point design. We collected and analyzed categories of both verbal and behavioural data in the two conditions. Our results show support for the co-dependent strategy and suggest ways that the codependent design can be used to support flexible collaboration on tangible tabletops for young adults.
\end{abstract}

\section{Author Keywords}

Tangible user interfaces; digital tabletop; interactive surfaces; codependent access points; collaboration; young adults.

\section{ACM Classification Keywords}

H.5.3 [Information interfaces and presentation]: Group and Organization; H.5.2. Information interfaces and presentation: User interfaces.

\section{INTRODUCTION}

Much research has been conducted to explore how to better support collaboration on digital tabletops. The large size of digital tabletops enables users to view and work on tasks together, which supports collaborative activities [23]. More recently, tangible user interfaces have been used in conjunction with digital tabletops to facilitate collaborative activity [25]. Using tangible objects on tabletops allows people to share, view, place and manipulate physical objects as tools and representations in collaborative activity. The physicality of objects has been shown to support awareness of each other's actions in collaborative activity [25].

Permission to make digital or hard copies of all or part of this work for personal or classroom use is granted without fee provided that copies are not made or distributed for profit or commercial advantage and that copies bear this notice and the full citation on the first page. To copy otherwise, or republish, to post on servers or to redistribute to lists, requires prior specific permission and/or a fee.

GROUP'14, November 06-09, 2014, Sanibel Island, Florida, USA.

Copyright is held by the owner/author(s). Publication rights licensed to ACM. ACM 978-1-4503-3043-5/14/11\$15.00.

http://dx.doi.org/10.1145/2660398.2660402
However, studies of tangible tabletop collaborative activity reveal disparate results $[17,20]$. For example, multiple physical access points offered by tangible tabletops have been shown to promote synchronous collaboration [25]. However, in another paper, researchers reported that this strategy resulted in parallel, independent work rather than collaborative activity [17, 21]. In order to support collaborative activity, some researchers from the collaborative learning field have suggested distributing information, skills, roles or tools among learners in a way that requires them to work together. This is called a collaboration jigsaw script [1]. Another approach is to hard-code system constraints (e.g. enforcing turn-taking) to force collaboration [19]. The drawback of these approaches is their inflexible nature. The challenge is to design tabletop systems that enable and encourage collaborative activity but do not enforce it $[1,13,21]$.

Antle and Wise suggest a variant of the jigsaw script that utilizes a system design that recognizes sequences of actions and involves a unique set of tangible input objects that can be split up and assigned to different users -- resulting in a system/physical/social configuration that either enables, encourages or enforces collaboration as the situation warrants [1]. In a system with codependent access points, inputs are sensed separately but processed together by the system $[1,13]$. That is, two or more input actions are required for a successful response. If the design also includes a unique set of tangible input objects, then the set can be split into groups and assigned to different users. The codependent access points enable users to collaborate by enacting sequences together. The assignment can be done to encourage (but not enforce) users to collaborate. Antle and Wise also propose that using tangible rather than touch-input objects reduces the chances of one user ignoring the assignments and taking over, or undoing another's actions because of social norms around object ownership and use (based on [24]). Taken together a set of unique codependently sensed, tangible, and user-assigned input objects may encourage flexible opportunities for collaboration. For brevity we call this approach co-dependent access (CD) and an unconstrained variation independent access (ID). Our research explores the strategy proposed by Antle and Wise. Our research questions are, (RQ1) Does a CD design on a tabletop encourage young adults to collaborate more than a similar design that is ID? (RQ2) What kinds of collaborative behaviours and interactional patterns emerge for each design strategy? Answering these questions will provide guidance for designers looking for alternative ways to encourage tabletop collaboration without enforcing it.

In order to address our questions, we conducted an exploratory, comparative study with 12 young adults who used a tangible, multi-touch tabletop application for collaborative land use 
planning, called Youtopia. In this paper, we present the results of our analysis comparing quantities and types (qualities) of verbal negotiation and physical collaboration in two conditions, which we call co-dependent (CD) and independent access (ID) points. We provide a summary of quantitative data, and then focus on a detailed analysis of the qualities of behaviours and interactions to better understand how our design strategies may facilitate collaborative activity. We discuss the implications of our results and propose four ways in which the $C D$ approach may be beneficial for the design of collaborative, tangible digital tabletop applications.

\section{RELATED WORK}

There is no one single definition of collaboration or collaborative activity. According to Goos et al. [10], collaboration is a reciprocal, coordinated interaction in which ideas and perspectives are explored and exchanged. Dillenbourg [7] views collaboration as a situation in which interaction and negotiation must happen between participants to successfully complete a task. This definition stands in contrast to cooperation, in which people may still work together to accomplish a task but negotiation and interaction are not necessary. Negotiation plays an essential role in collaboration [26]. Collaborative activity needs the negotiation not only of task-related content, but also of task structure in terms of roles, activities, and sub-task allocations [6]. Dillenbourg's definition also suggests the importance of equitable participation both verbal and physical -- for hands-on tasks. Equitable participation helps team members to better understand each other, adjust plans, and achieve the shared goals.

\subsection{Designing for Tabletop Collaboration}

Multi-touch and tangible digital tabletops have been suggested as one way of encouraging productive synchronous collaboration. However, the empirical findings of previous studies are contradictory $[17,21,24]$. Several studies suggest that multi-touch tabletops enable more synchronous collaboration than traditional user interfaces [22, 24]. Traditional computer technologies, such as a single mouse with PC, do not allow synchronous activity for multiple users [20]. The single-mouse situation forces users to share a single input and often results in frustration and reduced engagement $[14,22]$. In contrast, multi-touch tabletops enable multiple users to simultaneously engage in the same activity, which may simulate synchronous collaboration and avoid conflict over input controls (i.e., 'cursor wars'). For example, the multitouch system DiamondTouch [8] allows for synchronous collaboration among multiple users as well as multiple simultaneous touches from a single user. CollabDraw [18] uses cooperative gesture interaction techniques to support collaborative art and photo manipulation.

Benford et al. [4] present different approaches to interface designs that enable, encourage or enforce collaboration. Enabling collaboration refers to providing multiple access points that allow users to participate simultaneously. Encouraging collaboration refers to offering an incentive or functionality that encourages collaborative work. Enforcing collaboration refers to functionality that enforces specific collaborative actions, such as turn-taking. Encouraging collaboration is more proactive than only enabling collaboration, but not as inflexible as enforcement [17].

Combining tangible objects with multi-touch tabletop interaction may improve users' awareness of each other's actions and tool use $[12,24]$. For example, Speelpenning et al. [24] conducted an exploratory study to compare the differences between tangible and multi-touch tool use and the impact of tool use on collaboration in a digital tabletop game. Observational findings suggested that the physicality of the tangible tools facilitated individual ownership and announcement of tool use, which in turn supported awareness of each other's actions, and therefore more effective support for collaboration.

Tangible objects provide multiple access points to a tabletop application, which may lead to parallel rather than collaborative activity $[17,21]$. Several design strategies have been explored to avoid parallel use. For example, in a tabletop computing game called SIDES [19], turn-taking was used to regulate and ensure each individual's equitable participation in collaboration. However, this approach forces people to work together rather than encouraging them to collaborate, which results in less flexible collaboration.

The literature $[1,13,20]$ illustrates we do not yet understand how to reliably design tabletop systems that enable and encourage collaboration but do not force collaboration. Antle and Wise [1] suggest that positive interdependence, which will encourage collaboration, may be achieved through a combination of system, physical and social design. They suggest creating a system with co-dependent access points in which more than one input action must be taken in order to create a successful system response. Access points may be any potential input elements that enable users to interact and participate in a collaborative activity [11, 13]. They suggest using a unique set of tangible input objects - which may be tools or representations - that are essential to task(s) completion. Lastly, they suggest assigning the tangible objects to different users to encourage collaboration. If objects are not assigned (ID), the collaboration is enabled because the system still requires sequences of actions - which may be taken by one or more users. Conversely, authoritative assignment instructions (e.g. teacher in a classroom) can lean towards enforced collaboration (e.g. children are told not to exchange tangible objects). In this paper, we focus on the strategy that encourages equitable collaboration without restricting it. We implement Antle and Wise's approach for a land use planning activity to explore whether a CD design is more effective for encouraging collaboration than an ID design and to understand how these two design strategies effect collaboration in a tabletop activity for young adults.

\subsection{Analyzing Tabletop Collaboration}

Analysis of collaboration tends to focus on verbal and physical behaviours that people use to mediate collaborative activity [11, $15,20]$. The amount and type of explicit communication can indicate the degree of collaboration [9]. For example, verbal negotiation, such as talk or dialogue, plays an essential role in sharing mutual understanding among participants in face-to-face collaboration. Studies [11, 15] suggest various types of talk patterns are important in collaborative activities around tabletops. Jamil et al. [15] discuss how different tabletop designs lead to different talk patterns during collaborative activity. Similarly, Harris et al. [11] present results from a comparative study of multiple-touch and single-touch collaborative interaction on a tabletop activity, wherein a coding system of talk types was developed to measure the level of collaboration.

Physical interaction is also important in collaboration analysis. In a study presented by Hornecker et al. [12], they demonstrated that large surfaces provided users with opportunities to organize objects physically in space in order to support collaborative 
activity. The size of the surface also allowed each member to be visually aware of other members' activities.

\section{SYSTEM DESCRIPTION}

Youtopia is a collaborative, tangible, multi-touch tabletop sustainable land use planning activity (Figure 1). It was designed to support users to experience the challenges of sustainable land use planning. We implemented Antle and Wise's three tier (system/physical/social) design strategy. The system recognizes sequences of inputs made with a unique set of tangible stamps, which can be assigned in sets to each user, or left unassigned.

\subsection{Youtopia}

The system of Youtopia consists of a set of tangible stamps used for input onto a multi-touch tabletop map display (Figure 1). The main form of interaction with the map is through stamping land uses onto the map with the tangible stamps. There are two kinds of stamps: land uses and tools. Touch is used for basic system controls such as choosing a map or population size. A complete description of Youtopia can be found in [2]

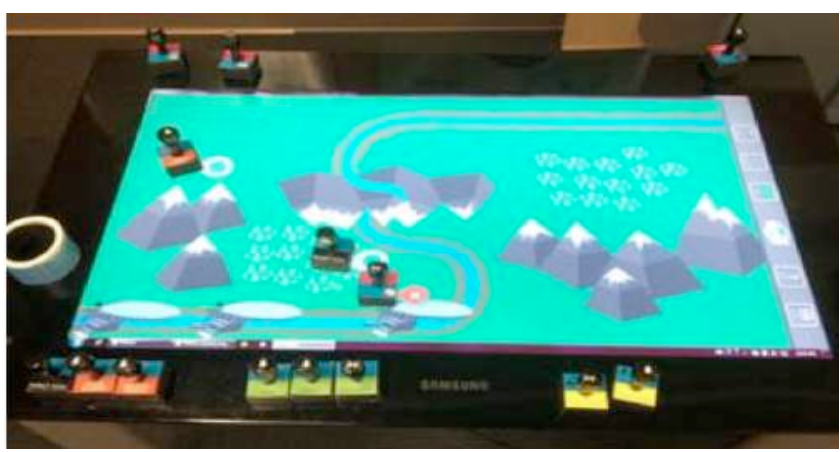

Figure 1. Youtopia: a collaborative hybrid tangible multitouch tabletop sustainable land use planning activity.

\subsubsection{Land use Stamps}

Land use stamps can be used either to designate natural resources as usable for human development or as development stamps to designate spaces for food, shelter or energy production facilities. Human developments cannot be built without first designating natural resources as usable. For example, in order to create a housing unit, a user must stamp the lumber stamp onto an area of forest to designate the lumber from the forest as usable. Then s/he can use the housing stamp to place a housing unit somewhere in the available grasslands (Figure 2). Youtopia requires codependent input: first lumber, then housing in order to create a shelter unit.



Figure 2. Stamping trees into lumber units.

\subsubsection{Tool Stamps}

A set of three additional stamps provide tool functionality, including erase, impact (which shows the current state of the world), and info (which shows information about each land use type). The eraser stamp undoes previous stamp actions. When placed anywhere on the map, the impact stamp displays an information overlay about the current state of the world in terms of the proportion of the population's needs being met for food, shelter and energy, as well as displaying the world's pollution level. Placing any stamp in the information ring displays a detailed information overlay about that stamp including what the land use is, which other stamps it is co-dependent on and what it produces. Users can rotate or scale the information overlay to share it using multi-touch. Both impact and information tools provide a freezing screen feature whereby all other functions are inactive when these stamps are in use (Figure 3).

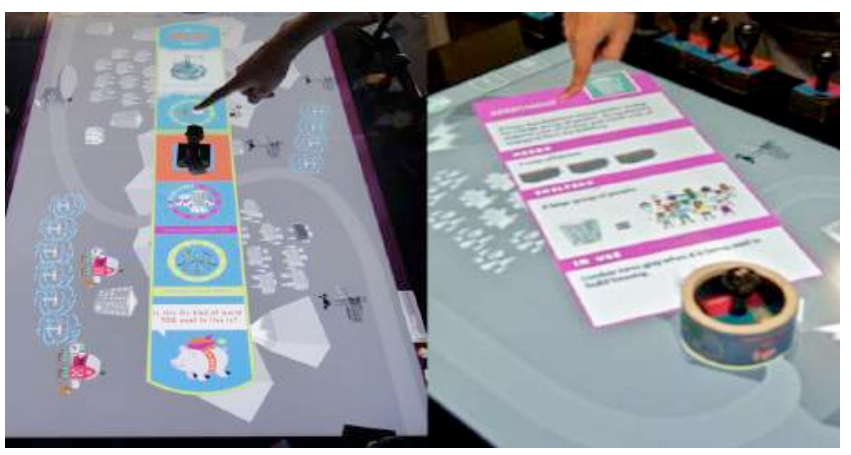

Figure 3. Impact tool and Information tool - freeze the display.

\subsection{Scenario of Use: Co-dependent and Independent Modes}

Youtopia can be used in two modes based on the instructions given to the users. In the co-dependent mode, the natural resource stamps (labelled with a tree on top of the handle) are given to one person assigned the role of natural resource planner, and the development stamps (labelled with a wrench) to another assigned the role of developer. In the independent mode, users do not have any roles and they can use any stamp.

The application begins with an undeveloped landscape that contains areas of natural resources (e.g. trees, river, coal reserves in mountains) and other "open" areas (e.g. grasslands). Participants use the stamps to designate what use will be made of each specific space on the interactive map. They can designate natural resources for preservation or use, and build food, shelter or energy sources to try to support either a small or large population's needs. The balance of preserving the natural environment while meeting the population's needs is up to the participants. Youtopia provides no explicit feedback on winning or losing in order to allow participants to explore options according to their values.

\section{STUDY METHODOLOGY 4.1 Study Design}

In our study we explore how the CD strategy compares to the ID strategy. We also explore how the two designs affect negotiation and decision making about the domain topic (sustainability). Youtopia only responds when resources are stamped before development stamps are used. In the co-dependent configuration (CD) we use this system rule AND social conventions to set up the 
condition where each person "owns" either resources or developments. Both users must act to create anything. This has the potential to set up conflict, which leads to the need to negotiate. In the independent group (ID), we remove the social constraint of assigning tools; this configuration represents a typical tabletop system in which a single user can take a series of actions with different objects to control interaction at any given time.

An exploratory comparative study was conducted. In the CD condition, pairs were asked to use their own stamp tools. In the ID condition, pairs could use any of the stamp tools. A withinsubjects design was used because group dynamics can influence collaboration [16]. To control for order effects, conditions were counterbalanced.

\subsection{Participants}

We collected data from 12 participants (four males and eight females) who played the activity in pairs. The participants were university students (aged 20 to 28). All participants had used a touch surface before (e.g. smart board, iPhone, iPod). Most participants had used a digital tabletop (eight of 12) and a TUI (eight of 12) before. However, none of them had played our system before. The groups were randomly assigned and participants knew each other to different degrees: some were classmates or friends (eight of 12) while others did not know each other at all (four of 12). Pairs in four groups were a male and a female while in two groups there were two females. Participants were rewarded with $\$ 5$ for participating in our study.

\subsection{Task}

The task was to "Create a world that you like to live in, which includes creating enough food, shelter and energy for a small population." The task was challenging because there were not enough resources to meet the needs of the population and keep the environment pristine. Users needed to discuss trade-offs, negotiate and use stamps and tools to designate land uses. This approach reflects typical planning activities in the real world. Participants used a different but equivalent map in the second condition to control for learning effects. In each condition, participants had 10 minutes for this task. The decision of 10 minutes was based on our previous experience of pilot studies. There was no fixed approach or "winning state" for the task. Participants could use different stamp tools to achieve the goal. For example, in order to make shelters for a small population, participants could use any combination of apartments, townhouses or single-family dwellings.

\subsection{Procedure}

Our land use planning application was set up on a Microsoft Surface table in a controlled lab space. The session began with a demographic survey. We gave participants a basic system tutorial. The pair then had five minutes to familiarize themselves with Youtopia. When they felt ready to begin, they were given the task. Each pair worked on the same task in each condition, changing conditions after 10 minutes. Post-task interviews of each participant were conducted after each of the two tasks. Sessions lasted about 30 minutes in total.

\subsection{Data Collection and Analysis}

Our mixed-methods approach involved collecting data including video (V.), structured observations (S.O.), system logs (S.L.), and post-interviews (P.I) in order to analyze verbal negotiation (V. \& S.O.), physical actions (V. \& S.L.), interactional patterns (V. \& S.O.) and participants' opinions (P.I.). We used quantitative methods to address our first research question (RQ1). Quantitative methods consisted of measuring the level (duration) and equity of verbal negotiation and level (duration) and equity of physical interaction in both conditions. We analyzed data with descriptive statistics depending on data type (median/range for ordinal, mean/standard deviation for interval). We used qualitative methods to address our second research question ( $R Q 2)$. Two researchers observed and recorded different types of verbal negotiation and physical actions, identified interactional patterns, and asked for participant's opinions about their collaborative work after each condition.

\subsubsection{Level of verbal negotiation}

Level of verbal negotiation refers to the amount of task-related utterances that either participant made during the task session [11]. To avoid the challenges of time-consuming video coding which are not warranted by an exploratory study, we used structured observational sheets with four options to categorize the level of each pair's utterances (none, few, some, many). One researcher observed each participant, collected categorical utterance data which was then summed for the pair, and assigned a value 0 (none) to 4 (many/a lot). For example, if the pair did not talk in the entire gameplay, then 'none' was chosen. If they talked almost constantly to each other about the task - for at least 7.5/10 minutes - then 'many' was chosen. While we lose precision with our approach, we can easily and reliably identify large differences between groups, which is a suitable approach for an exploratory study.

\subsubsection{Equity of verbal participation}

Equity of verbal participation refers to the differences in the duration of utterances between two participants during the task session [11]. The equity of utterances can reflect the degree of an individual's participation. We calculated the difference of the duration in terms of each participant's utterances. If the difference was less than approximately two minutes (10\%), it was considered most equitable. If the difference was more than five minutes, then we considered it unequal. Categorical data about equitable participation (unequal, some equity, most equity) was collected for each task in each session. We then coded this data from 1 to 3 , with 3 being most equitable.

\subsubsection{Level of physical interaction}

Level of physical interaction refers to the total number of touches and tangible object uses during the task session. System logs were used to record interval data including the total number of stamp uses; tool uses (eraser, impact and information tool); touches on feedback tabs; and touches on the impact tool display.

\subsubsection{Equity of physical participation}

Data sets from system logs could not indicate which participant took each action. We used a video camera to record participants' actions and distinguish each participant's stamping actions and touching actions based on both video and system log datasets. By counting the number of stamping and touching actions per participant, we could compare two participants' frequency of physical participation in each condition.

\subsubsection{Types of verbal negotiation}

We were also interested in the content of verbal utterances about the task as well as the similarities and differences between the CD and ID conditions. Researchers iteratively developed a coding scheme of types of verbal negotiations based on the literature review $[11,15]$ and pilot studies before the experiment. The final categories were: 
1. Task-focused discussion and negotiation (e.g., strategies)

2. Information exchange (e.g. instructions)

3. Conflict dialogues (e.g. disagreement)

Structured observational sheets and video data were used to collect the number of instances of each types of utterance and write descriptive notes about when they occurred.

\subsubsection{Type of physical action}

Type of physical interaction refers to different physical patterns that emerged during system use. We focused on physical actions between participants. The coding themes were developed prior to the experiment [11]. The main patterns of physical interaction were:

1.Purposeful actions or gestures (e.g. pointing to a place on the map, passing tools to each other)

2.Contents sharing through tools and multi-touch (e.g. rotating or scaling contents for the other)

3.Conflict over use of tools (e.g. grab tools at the same time)

We analyzed which type of physical actions emerged during the CD and ID conditions and how participants used these actions during collaboration in the different conditions.

\subsubsection{Interactional patterns}

Interactional patterns refer to how participants worked with each other during the task. We hypothesized that co-dependent access points might support collaborative rather than parallel work. We were interested in the interactional patterns that participants developed to coordinate their work in tasks. We used observational notes, video data and post-interviews (with one open-ended question for each participant: How do you think the different set-ups impacted your collaboration?) to help us understand interaction.

\section{RESULTS}

Our results provide insight into the similarities and differences in collaborative behaviours between the $\mathrm{CD}$ and ID conditions. Quantitative results provide information about levels of verbal negotiation and physical interaction while qualitative findings reveal types of verbal negotiation, physical interaction and working strategies.

\subsection{Level and Equity of Verbal Negotiation}

There was no difference in verbal negotiation between two conditions (Table 1). However, we noticed that when participants started parallel work in the ID condition they stopped verbal negotiation, perhaps because they did not need group awareness for independent work.

More pairs in the $\mathrm{CD}$ condition participated equally in verbal negotiation than in the ID condition (Table 1). Participants in both conditions usually had turn-taking talk patterns. Yet we found the duration of their verbal utterances was different. Since participants had their own roles and tools in the CD condition, they had to discuss with the partner in order to complete tasks. As shown in the following excerpt, we found that co-dependent use of tools promoted more equitable contributions.

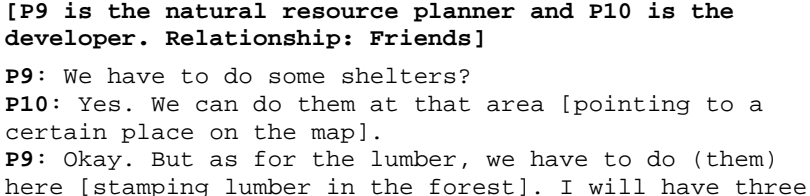

for, for...

P10: for a townhouse [stamping a townhouse on the map]

In the ID condition, we often observed that one participant played a "dominant role" by proposing strategies or offering information to the other participant. Conversely, the non-dominant participant often asked the dominant person for suggestions or confirmations in the decision-making process. The "no roles" configuration decreased the equitable participation from both players.

\begin{tabular}{|c|c|c|}
\hline & $\begin{array}{c}\text { CD Median } \\
\text { (Range) }\end{array}$ & $\begin{array}{c}\text { ID Median } \\
\text { (Range) }\end{array}$ \\
\hline $\begin{array}{c}\text { Level of verbal negotiation } \\
\text { (median level per session) }\end{array}$ & $4(2)$ & $4(3)$ \\
\hline $\begin{array}{c}\text { Equity of verbal participation } \\
\text { (median level per session }\end{array}$ & $3(1)$ & $2(1)$ \\
\hline
\end{tabular}

Table1. Level (0-4) of verbal negotiation and participation

\subsection{Level and Equity of Physical Interaction}

The mean number of physical interactions in the $\mathrm{CD}$ condition was a little higher than in the ID condition. We also found that pairs used the impact tool more often in the CD condition than in the ID condition (Table 2). It is possible that encouraging codependent use of tools may make pairs focus more on checking progress as part of their world-building strategy.

\begin{tabular}{|c|c|c|}
\hline & $\begin{array}{c}\text { CD: } \\
\text { Mean (SD) }\end{array}$ & $\begin{array}{c}\text { ID: } \\
\text { Mean (SD) }\end{array}$ \\
\hline $\begin{array}{c}\text { Level of physical interaction } \\
\text { (mean \# events per session) }\end{array}$ & $94(24.1)$ & $85(14.9)$ \\
\hline $\begin{array}{c}\text { Level of the impact tool use } \\
\text { (mean \#uses per session) }\end{array}$ & $10(8.2)$ & $6(5.2)$ \\
\hline
\end{tabular}

Table 2. Level of physical interaction and impact tool use

The equity of physical participation between pairs in the CD condition was much better than that in the ID condition (Table 3 ). The results were also consistent with our observational findings. In the ID condition we found that it was common for one participant to conduct all the actions while the other only offered verbal suggestions without physical involvement. An example is presented below:

[No role. Relationship: Strangers]

P2: What do you want, houses or townhouses?

P1: Houses. We need houses, but probably not close to

here [pointing to the hydro dam] because... Why don't we

move the hydro to here [erasing the hydro in the center

and rebuilding it on the edge of the map].

P2: Okay.

P1: Far away (from the forest).

P2: Now where do you want to build the house?

P1: Both these areas will be fine. Here [pointing to the forest] or here [pointing to grasslands close to forest].

\begin{tabular}{|c|c|c|c|c|}
\hline & \multicolumn{2}{|c|}{ CD: Mean (SD) } & \multicolumn{2}{c|}{ ID: Mean (SD) } \\
\hline $\begin{array}{c}\text { Equity of physical } \\
\text { participation } \\
\text { (mean \# events per } \\
\text { session) }\end{array}$ & P1 & P2 & P1 & P2 \\
\cline { 2 - 5 } & $\begin{array}{c}50 \\
(12.4)\end{array}$ & $\begin{array}{c}44 \\
(17.0)\end{array}$ & $\begin{array}{c}50 \\
(14.7)\end{array}$ & $\begin{array}{c}35 \\
(13)\end{array}$ \\
\hline
\end{tabular}

Table 3. Equity of physical participation between players 


\subsection{Types of Verbal Negotiation}

\subsubsection{Task focused negotiation}

People in both CD and ID conditions spent a large amount of time discussing their vision and strategies. The most common themes were what kind of a world they intended to have (the overall vision) and how specifically they planned to build it (their strategies). Although people in both conditions talked about their tasks and strategies, we found there was a slight difference in the ways they spoke about them. In the CD condition, we noticed that in three of six groups both players used declarative sentences to state their opinions. Participants were more deliberative about their decisions when they controlled their own tools, which gave their contributions more equal weight in the decision-making process.

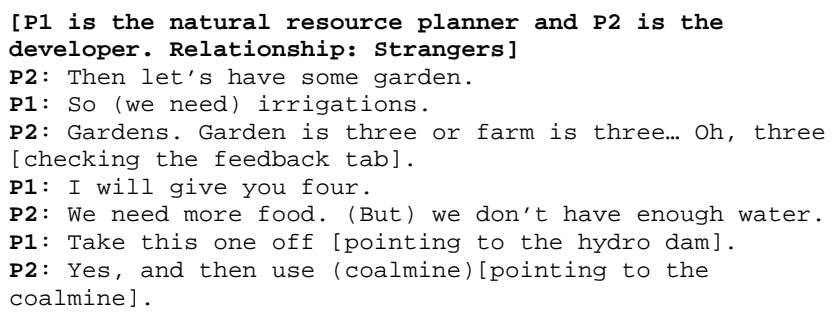

In contrast, when people (three out of six) worked together in the ID condition, they tended to ask for confirmation first before conducting the next action. It was common to see a non-dominant participant ask the other for suggestions in the decision-making process. Similarly, when the dominant participant attempted to make any movement, he/she also informed the other one. Actions were more tentative.

[No role. P1: dominant role P2: non-dominant role] P1: As for the energy, let's do hydro because it is clean enough. Coalmine [pointing to the stamp] is not clean. Right?

P2: Okay.

P1: We probably do here [pointing to a certain place on the map]?

P2: Yes.

P1: Hydro can be built only on the river [reading the feedback tab for P1].

P2: And, then, we need house. You want the house or the townhouse?

P1: Probably house. We want [to] build a house. Maybe not close to here [hydro].

P2: Yeah.

\subsubsection{Information exchange}

We observed that participants sometimes exchanged information or taught each other about how to use Youtopia in both conditions. Compared to the task-focused discussion, there was a low level of discussion related to information exchange. We did not observe any obvious difference between two conditions. Instead, order affected the levels of information exchange. Most of the information exchange occurred during the first session of the experiment. There were several types of information exchange. The most common way was directly asking. If one participant had doubts or concerns, they simply proposed questions to the other player. We also found that people used "reading aloud" to exchange information. For example, if they were reading the texts on feedback tabs, they often read it aloud (Figure 4). When participants intended to make an action, they tended to verbalize it first.

\subsubsection{Conflict}

We found that two groups had some conflict in the CD condition, while none occurred in the ID condition. The conflict stemmed from their different thoughts about how to make decisions based on their own roles. In part, this may be because participants were more dedicated to their own roles in the CD condition, which encouraged more negotiation. Sometimes the negotiations involved conflict, which was not always resolved.
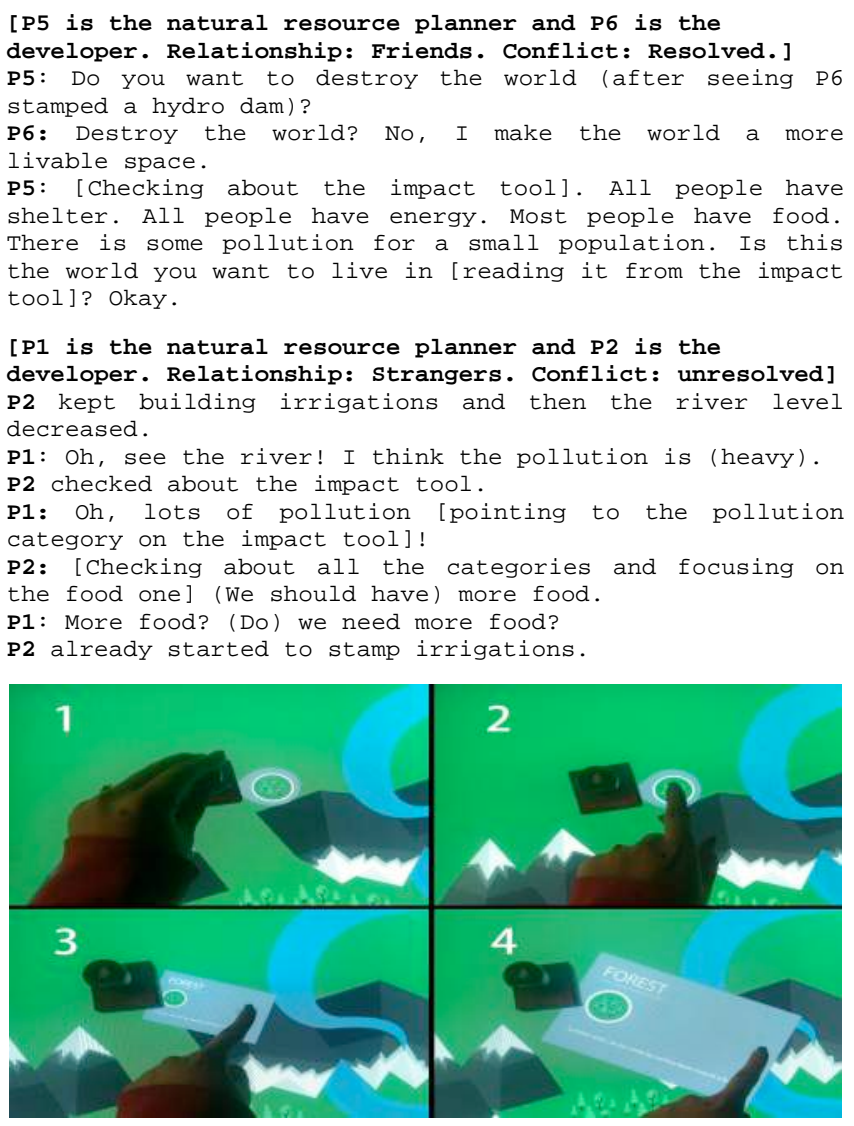

Figure 4. Dragging feedback tab reveals error massage.

\subsection{Types of Physical Action}

\subsubsection{Purposeful actions and gestures}

Participants in both CD and ID conditions used many pointing gestures to indicate a particular place on the map or a specific stamp on the table. We observed that people used more pointing gestures in the $\mathrm{CD}$ condition than in the ID condition. When one participant attempted to create a unit at a particular place on the map, they often informed the other participant through verbal sentences with a pointing gesture.

[No role. Relationship: Strangers]

P1: let's do (here) [pointing to a pace on the grass]. P2: yes [pointing to the same place]!

We found there were several factors that seemed to be related to people to use pointing gestures. The most common was the use of the impact tool. It was common for participants to point to each category of the world state display (shelter, food, energy, pollution) when discussing their strategies (Figure 5). Pairs used the impact tool 62 times in the CD condition, while it was used only 39 times in the ID condition, which influenced the levels of pointing gestures. 
Another common use of pointing gestures was to indicate a particular place on the map. We observed this behaviour in both conditions. Participants often used it when giving suggestions or discussing strategies. In the ID condition, there were many gestures related to participants managing and sorting stamps together. In contrast, the role assignment in the $\mathrm{CD}$ condition contributed to less need to organize and manage tools. This is a secondary advantage of the CD strategy - it enables users to focus less on tool management and more on the task at hand.

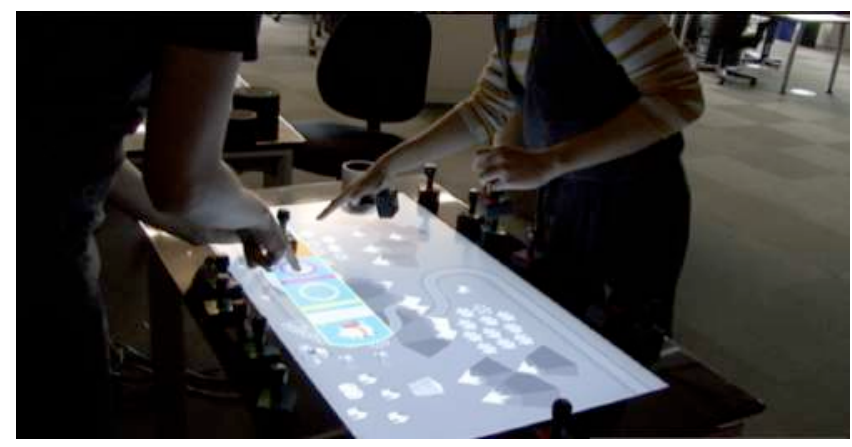

Figure 5. Pointing to the category of the world state display (impact tool) when discussing strategies.

\subsubsection{Content sharing through tools}

In Youtopia, the impact and information tools provide a freezing screen feature whereby all other functions are inactive when these stamps are in use.

It is important to note that people started to concentrate on the same content and discussed their strategies when using the impact and information tools (Figure 6). We infer that the freezing feature might provide external tensions that force people to share group awareness.

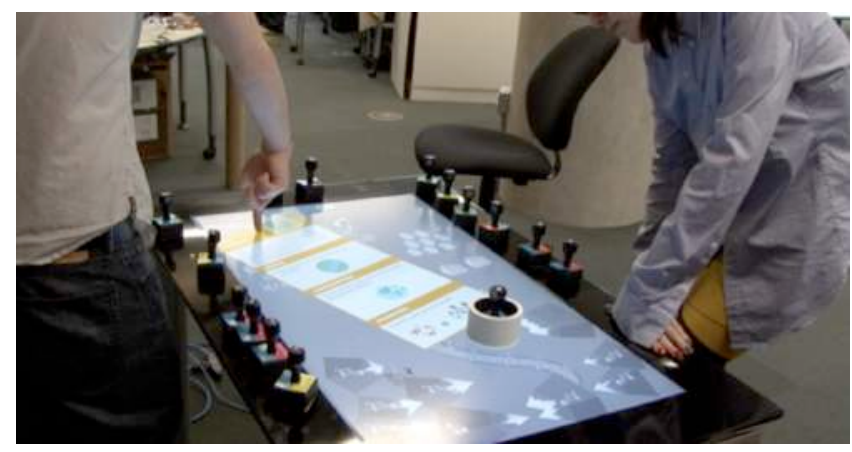

Figure 6. Sharing content through the Information tool.

\subsubsection{Conflicting uses of tools and space}

We observed conflicting uses of tools in both CD and ID conditions, but the types of conflicts were completely different. In the ID condition, players reached for the same stamp by accident (Figure 7). When they realized it, they usually let the other use it first. We suggest that this is due to a strong social constraint in young adults about not taking objects out of another person's hands (as found in [24]). However, in the CD condition, when one group turned their collaboration into competition, we observed them intentionally stealing the other's tools and using them as a part of their task.

[P5 is the natural resource planner and P6 is the developer. Relationship: Friends]

P6: My job is to destroy the world. You see.

P5: I don't want you to build the factories here [holding the eraser tool to erase factories]. Why not (to do something else)?

P6: That's the point! This game for me is to create the pollution. Ha-ha!

P5: No! You destroy the world!

P6: I really want to do this [directly picking up P5's lumber stamp to use it].

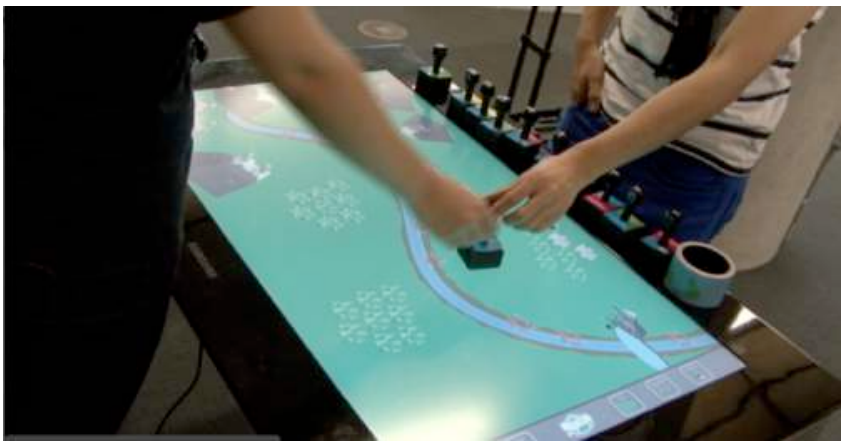

Figure 7. Reaching the same stamp in the ID condition.

Conflict over use of space only occurred during parallel work in the ID condition. We noticed that sometimes the two participants would use their stamps at the same place on the map or their actions would impact the other's actions (Figure 8). For example, when one participant (P2) was creating houses (lumber->house) and the other participant (P1) was creating gardens (irrigation>garden) in the ID condition, they had some conflict over use of space on the map.

[No role. Relationship: Strangers]

P2 created a house close the irrigation.

P1 kept trying to stamp the garden but fails.

P1: Sorry. It is too close to (my irrigation) [pointing to

the house that P1 just created]. Where is the eraser tool? I have to erase (it).

P2: Okay. It doesn't matter.

P1: You can build it here [pointing to another place which is a little bit offset from the original place].

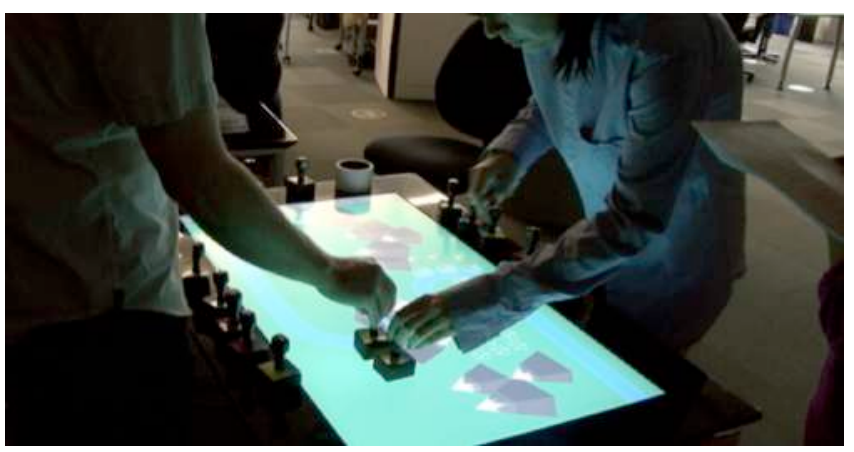

Figure 8. Conflict over use of space in the ID condition.

We found that the conflict over use of space was an effective trigger point for people to switch from parallel work to collaborative or cooperative modes. It significantly encouraged people to start verbal negotiation and physical interaction.

\subsection{Interactional Patterns}

There was a significant difference in the interactional patterns between the $\mathrm{CD}$ and ID conditions. We identified four main patterns: (a) collaboration with shared goals and co-dependent uses of tools; (b) collaboration with shared goals but with only one person executing actions; (c) cooperation with separate sub-tasks and parallel uses of tools; and (d) no collaboration or cooperation with parallel uses of tools. 


\subsubsection{Co-dependent condition}

The dominant interactional pattern in the $\mathrm{CD}$ condition was collaboration with co-dependent use of tools. In this case, participants discussed and performed the task together. Five of six groups adopted this strategy during the whole process.

Similar behaviours were found in the five groups that adopted the actively collaborative pattern. They first talked with each other about which units they wanted to create, why they had to create them as well as how or where to build on the map. Then each participant manipulated their own stamp to create a specific unit.

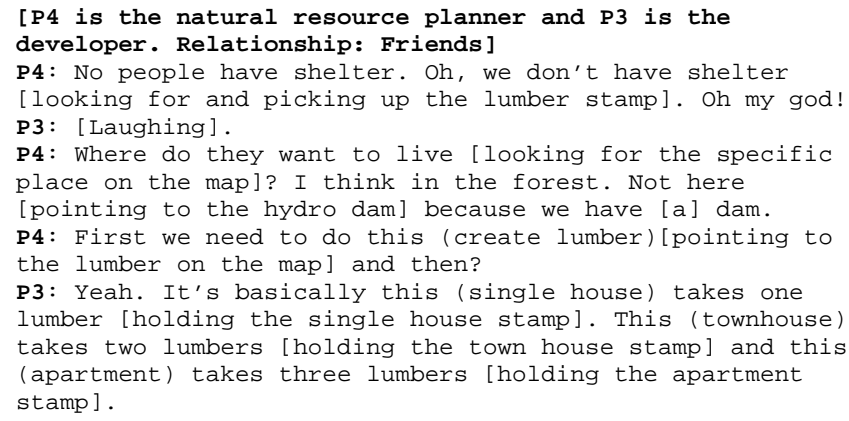

Rather than actively discussing strategies, we observed that participants in one group (out of six) only asked for basic help from each other in order to complete their stamping actions during the whole activity.

[P11 is the natural resource planner and P12 is the developer. Relationship: Strangers. They did not have any conversation for almost three minutes before the following talk]

P11: Oh, I can't have a farm because I need two these (irrigations).

P12: You need more these irrigations?

P11: Yes.

P12: [Stamping two irrigations for P12].

Another interesting observation in the CD condition was that in one group, one participant stole tools from the other during the activity, which caused conflict over the tools. P6 who acted as the developer stole tools from P5 who was the natural resource planner. However, the independent uses of tool did not lead to subsequent parallel work. They still continued to verbally argue and negotiate with each other.

\subsubsection{Independent condition}

Compared to the $\mathrm{CD}$ condition, there was no single dominant interactional pattern in the ID condition. Participants appeared to adjust their interactional patterns from time to time. In general, we observed three patterns in the ID condition. In the first pattern, pairs cooperated to perform tasks with parallel uses of tools. They first broke down the task and assigned different sub-tasks to each person. Then, each person focused on his or her own part with parallel uses of tools to achieve their shared goals. We observed this pattern in two groups.

[No role. Relationship: Strangers]

P1: Let's do houses here [pointing to a place close to forests].

P2: We are going to cut these trees [holding both lumber and house stamps to create a single house].

P1: I will sow some more gardens [picking up both

irrigation and garden stamps to build gardens].

A second pattern involved pairs working in parallel but not coordinating their activity beforehand. The independent use of tools made it possible for each person to concentrate his/her own subtasks. In one group, participants worked individually with little verbal or physical interaction during the whole activity.

A third pattern involved one person physically controlling and using the stamps, while the other person passively watched or offered verbal suggestions. Both participants were involved in verbal collaboration, but only one participant physically manipulated tools and executed tasks. Two (out of six) groups interacted this way many times in their collaboration.

In the ID condition, most groups had two or three interactional patterns, with one dominant pattern. They switched between patterns several times during the activity. Based on observation, we found three possible motivations for their switches: (a) conflicting use of space on the map; (b) frozen screen; and (c) familiarity with the system and activity.

\subsubsection{Post-interviews: experiences between two conditions}

Participants responded in a variety of ways to the post-task question: How do you think the different set-ups impacted your collaboration? One group reported that they really enjoyed learning together in the $\mathrm{CD}$ condition. They also indicated that the CD use of tool made them feel like a team. Two other groups reported that they preferred the ID use of tools because it made their collaborative work more efficient and gave them more freedom to explore different tools. Three groups mentioned that they preferred to use the CD configuration early while learning about the activity. They said that after becoming familiar with the activity, they would like to switch to the ID configuration because it gave them more freedom and ability to explore.

\section{LIMITATIONS}

We do not make strong claims in this exploratory study for several reasons. First, we have a small number of participants, which is suitable to an exploratory study with detailed qualitative analysis, but limits generalization. We categorized our quantitative data coarsely, which means we may not have picked up small effects. In addition, four groups of the pairs knew each other while the other two groups of pairs were strangers. This difference typically influences the dynamics of collaboration. Participants also had different experiences using tangible and multi-touch technologies. Although participants were given time to explore Youtopia and familiarize themselves with it, we found there was still a learning curve for a few participants, which may have contributed to inequitable participation. While we used two observers for data collection, we did not do a detailed inter-rater analysis for coding video data for counts of individual's physical actions so we use this data cautiously.

\section{DISCUSSION}

Our results provide some evidence that the CD design strategy encouraged more equitable verbal participation and physical interaction compared to the ID strategy. Our work is consistent with findings that having multiple input objects rather than single objects reduces discussion about tool organization or turn-taking (e.g. [11]), However, we have the best of both worlds in that users have their own tool set and a few shared tools that can be used to synchronize activity. The design of a CD system enables collaboration. By using physical grouping and social context (object assignment/instructions) the nature of the interaction can be changed in real time. Thus an additional benefit of the CD approach is that it is more flexible for encouraging collaborative activity because it can be adapted in real time to suit the dynamics 
of the group, task or context. The analysis of video, observational and interview data led us to suggest four ways the CD design can be used to encourage flexible collaboration.

\subsection{Supporting Different Group Dynamics}

We hypothesized that people would work independently without collaborating in the ID condition. Our results indicated that some pairs did just that. However, pairs also enacted other interactional patterns, such as cooperatively splitting up work into separate tasks. The flexible nature of the $\mathrm{CD}$ design means that a group or group leader could decide to use a cooperative "divide and conquer" approach simply by reassigning the input objects or roles. Thus, our CD approach can be modified to support different group dynamics and strategies as needed. On the other hand, if one or more users are not participating then they can be assigned tools or roles to encourage more equitable verbal and physical participation. Our approach is consistent with findings in [20] that suggest hybrid digital-physical interfaces enable equitable participation. The CD approach to digital-physical tabletops may be particularly useful in contexts where participation by all group members is paramount (e.g. learning, community land use planning).

\subsection{Supporting Productive Conflict}

The CD groups had more conflict over ideas (rather than tools) which can be beneficial in eliciting each user's values around land use priorities, and lead to negotiation and compromise necessary in land use planning. Thus, in a situation that requires conflict to elicit values, or conflict to learn or negotiate or trigger reflection, shifting the object assignment to a CD strategy will likely encourage productive conflict. The ID group exhibited more evidence of non-collaborative behaviours involving nonproductive conflict (e.g. dominance) and parallel activity. In these cases the group leader or instructor or possibly even the system could suggest or mandate shift to a CD mode through object assignment. The design of a unique set of tangible input objects which are recognized individually but processed by the system codependently creates a system that encourages productive conflict over the domain or activity rather than unproductive conflict over input or tool use. This relation between interaction technique and the nature of productive dialogue about the application domain versus application itself has been noted by others (e.g. [15]).

\subsection{Supporting Different Phases of Interaction}

Our post-task interview revealed that people use different patterns of interaction at different points in the task. At the beginning, people may need to learn and explore Youtopia together so they can also learn from each other, scaffolding or accelerating their learning process (as suggested in [10]). However, as people become familiar with the system and tools, they may want to interact more independently depending on their goals, ideas, personalities and strategies. Much previous work has treated collaborative activity as an all-or-nothing phenomenon. We suggest that the CD strategy, which can be used to support different ways of interacting, and can be configured in real time, may be more beneficial than strategies (e.g. hard coding turn taking (e.g. [19]) or roles (e.g. [3]) or tasks (e.g. [7,18]) that enforce collaboration throughout an activity. For example, at the beginning of a session, participants may be instructed (by a facilitator, teacher or the system) to take on roles to support the CD mode and encourage equitable learning participation. Later on in the session, participants could be enabled to drop their roles and switch to the ID mode to enable independent work or divide-andconquer approaches, and then later come back together to further collaborate. However, if collaboration is desired, then the CD mode can remain intact for the duration of the task.

\subsection{Supporting Shared Check in Points}

An additional feature of our system that we have found beneficial is when various tools disabled interaction and displayed an overlay on the map. Since the Information and Impact tools were not assigned to any one user, any user can decide its time to "check in" and create a shared check in point. We found that our system promoted the kind of group sharing and awareness that is essential to collaborative work through this functionality. By disabling interaction when one participant displays important information, the other participant is encouraged to attend to that information and possibly discuss it with his/her partner. This enables participants to maintain a shared awareness and helps them to coordinate their subsequent interactional patterns. We observed that in both conditions when pairs employed the impact or information tools, they tended to talk and work together after they resumed interaction with Youtopia. We suggest this might be more beneficial in the ID as a means to get team members to "check in" with each other. This observation is consistent with results from the work on a tabletop game called Futura [3]. We suggest that freezing the map screen to display important information enables that information to act as a referential anchor [5]. The map and information provide a common reference that anchors the participants' attention to a shared representation of the world state or other important information. Letting any user use a check in tool or alternatively giving each user such a tool is another important way that the system and social design can enable effective collaboration. Although this feature is unrelated to the CD strategy, it is complementary and enhances the benefits of this approach.

\section{CONCLUSION}

We present the results of an exploratory study that compared the similarities and differences in collaborative behaviours of young adults between CD and ID design strategies for a tangible tabletop activity. We found that the CD design supported more equitable verbal and physical participation. It encouraged participants to discuss their goals and decisions. The ID design sometimes led to parallel interaction. It also enabled a variety of working strategies and purposeful gestures. In both cases, freezing the display with informational tools encouraged subsequent collaborative behaviours. Overall, our results support the benefits of using the $\mathrm{CD}$ approach to support collaboration. We also found secondary benefits in that $\mathrm{CD}$ can be adapted easily, through social context, to be responsive to group dynamics, different goals around productive conflict, task phases and working approaches. We suggest that this approach will be applicable to any tabletop system in which multiple inputs can be processed co-dependently and input objects can be divided into unique groups. Further research is needed to test out these claims with other tabletop applications.

\section{ACNOWLEDGMENTS}

Thanks to NSERC, SSHRC, PICS and the GRAND NCE for funding to pursue this project. Thanks to Rachael Eckersley, Saba Nowroozi, Perry Tan, Amanda Willis, Jillian Warren and Allen Bevans for the design of Youtopia. 


\section{REFERENCES}

[1] Antle, A. N. and Wise, A.F. (2013). Getting down to details: Using learning theory to inform tangibles research and design for children. Interacting with Computers 25, 1. 1-20.

[2] Antle, A. N., Wise, A.F., Hall, A., Nowroozi, S., Tan, P., Warren, J., Eckersley, R., Fan, M. (2013). Youtopia: a collaborative, tangible, multi-touch, sustainability learning activity. In Proceedings of the $12^{\text {th }}$ International Conference on Interaction Design and Children (IDC), ACM. 565-568.

[3] Antle, A. N. Tanenbaum, J., Bevans, A., Seaborn, K., and Wang, S. (2011). Balancing Art: Enabling public engagement with sustainability issues through a multi-touch tabletop collaborative game. In P. Campos, N. Graham, J. Jorge, N. Nunes, P. Palanque, M. Winckler (Eds.) INTERACT 2011Lecture Notes in Computer Science, vol 6947, Springer Berlin/Heidelberg. 194-211.

[4] Benford, S., Bederson, B., Akesson, K., Bayon, V., Druin,A., Hansson, P., \&Taxen ,G. (2000, April). Designing storytelling technologies to encourage collaboration between young children. In Proceedings of the SIGCHI Conference on Human Factors in Computing Systems (CHI), ACM. 556-563.

[5] Clark, H.H., Brennan, S.E. (1991). Grounding in communication. In Perspectives on Socially Shared Cognition, American Psychological Association, Washington, DC. 127-149.

[6] Churchill, E. F., \& Snowdon, D. (1998). Collaborative virtual environments: an introductory review of issues and systems. Virtual Reality, 3(1). 3-15.

[7] Dillenbourg, P. (1999). What do you mean by collaborative learning?. Collaborative-learning: Cognitive and Computational Approaches. Oxford: Elsevier. 1-19.

[8] Deitz, P. and Leigh, D. (2001). DiamondTouch: A MultiUser Touch Technology. In Proceedings of the $14^{\text {th }}$ annual ACM symposium on User Interface Software and Technology (UIST), ACM. 219- 226.

[9] Gutwin, C., \& Greenberg, S. (2000). The Mechanics of Collaboration: Developing Low Cost Usability Evaluation Methods for Shared Workspaces. In IEEE Workshop on Enabling Technologies: Infrastructure for Collaborative Enterprises (WETICE 2000). Proceedings. IEEE $9^{\text {th }}$ International workshops on. 98-103.

[10] Goos, M., Galbraith, P., \& Renshaw, P. (2002). Socially mediated metacognition: Creating collaborative zones of proximal development in small group problem solving. Educational Studies in Mathematics, 49, 193-223.

[11] Harris, A., Rick, J., Bonnett, V., Yuill, N., Fleck, R, Marshall, P, \& Rogers, Y. (2009). Around the table: Are multiple-touch surfaces better than single-touch for children's collaborative interactions? In Proceedings of the $9^{\text {th }}$ international conference on Computer Supported Collaborative Learning (CSCL)- vol 1. International Society of the Learning Sciences. 335-344.

[12] Hornecker, E., Buur, J. (2006). Getting a grip on tangible interaction: A framework on physical space and social interactions. In Proceedings of CHI'06, ACM. 437-446.

[13] Hornecker, E., Marshall, P., \& Rogers, Y. (2007). From entry to access: how shareability comes about. In Proceedings of Designing pleasurable products and interfaces, ACM. 328342 .
[14] Inkpen, K., Booth, K.S., Klawe, M., Upitis, R. (1995). Playing together beats playing apart, especially for girls. In CSCL'95, L.Erlbaum Associates Inc. 177-181.

[15] Jamil I., O’Hara, K., Perry, M., Karnik, A., \& Subramanian, S. (2011). The Effects of Interaction Techniques on Talk Patterns in Collaborative Peer Learning around Interactive Tables. In Proceedings of CHI'11, ACM. 3043-3052.

[16] Meerbeek, B., Bingley, P., Rijnen, W., Hoven van den, E.Pipet. (2010). A Design Concept Supporting Photo Sharing. NordiCHI'10, Reykjavik, Iceland.335-342.

[17] Marshall.P., Hornecker, E., Morris, R., Sheep Dalton, N., \& Rogers, Y. (2008).When the fingers do the talking: a study of group participation with varying constraints to a tabletop interface. In Horizontal Interactive Human Computer Systems, TABLETOP 2008, $3^{\text {rd }}$ IEEE International Workshop. 33-40.

[18] Morris, M. R., Huang, A., Paepcke, A., \& Winograd, T. (2006). Cooperative gestures: multi-user gestural interactions for co-located groupware. In Proceedings of CHI'06, ACM. 1201-1210.

[19] Piper, A. M O'Brien, E., Morris, M.R. \& Winograd, T. (2006). SIDES: A cooperative tabletop computer game for social skills development. In Proceedings of the $20^{\text {th }}$ anniversary conference on Computer Supported Cooperative Work (CSCW). Banff, Canada: ACM. 1-10.

[20] Rogers, Y., Lim, Y., Hazlewood, W., Marshall, P. (2009). Equal Opportunities: Do Shareable Interfaces Promote More Group Participation Than Single User Displays? Human Computer Interaction 24(1/2). 79-116.

[21] Stanton, D., \& Neale, H. (2003). The effect of multiple mice on children's talk and interaction. Journal of Computer Assisted Learning, 19(2). 229-238.

[22] Stewart, J.,Raybourn, E.M, Bederson, B., \&Druin, A.(1998). When Two Hands are Better Than One. In Proceedings of CHI'98, ACM. 287-288.

[23] Subramanian S., Pinelle D., Korst J., Buil V.(2007). Tabletop collaboration through tangible interactions. Enabling Technologies: Infrastructure for Collaborative Enterprises'07. WETICE' 07. 16 ${ }^{\text {th }}$ IEEE International Workshop. 412-417.

[24] Speelpenning, T., Antle N. A., Doering, T. and van den Hoven, E. (2011). Exploring how tangible tools enable collaboration in a multi-touch tabletop game. In HumanComputer Interaction-INTERACT'11. Springer Berlin Heidelberg, 605-621.

[25] Waldner, M., Hauber J., Zauner J., Haller M., Billinghurst M. (2006). Tangible Tiles: Design and Evaluation of a Tangible User Interface in a Collaborative Tabletop Setup. In Proceedings of the $18^{\text {th }}$ Australia conference on ComputerHuman Interaction: Design: Activities Artefacts and Environments, ACM. 151-158.

[26] Wardhaugh, R (1985). How Conversation Works, Basil Blackwell, USA. 1-230. 